\title{
Wireless Energy Transfer using Zero Bias Schottky Diodes Rectenna Structures
}

\author{
Vlad Marian $^{1, \mathrm{a}}$, Salah-Eddine Adami ${ }^{1, \mathrm{~b}}$, Christian Vollaire ${ }^{1, \mathrm{c}}$, Bruno Allard $^{1, \mathrm{~d}}$ \\ Jacques Verdier ${ }^{2, e}$
}

${ }^{1}$ Université de Lyon, Ecole Centrale Lyon, INSA Lyon, Laboratoire Ampère, CNRS UMR 5005, F-69134 Ecully Cedex, France
${ }^{2}$ Institut de Nanotechnologies de Lyon (INL) - CNRS UMR 5270, INSA Lyon, F-69621, Villeurbanne, France
avlad.marian@ec-lyon.fr, ${ }^{b}$ salah-eddine.adami@ec-lyon.fr, cchristian.vollaire@ec-lyon.fr, dbruno.allard@insa-lyon.fr, ejacques.verdier@insa-lyon.fr

Keywords: rectenna; wireless energy transfer; energy harvesting; low power; MPPT

\begin{abstract}
This paper presents several RF-to-DC converter topologies and compares their performances based on measurements made on fabricated prototypes. A medium range wireless low power transmission experiment is presented. A low power DC-DC boost converter designed rectenna-generated energy conditioning is also discussed.
\end{abstract}

\section{Introduction}

Wireless energy transfer is the process of sending electrical energy from one point to another without the use of a conducting environment. This can be done using two different approaches. The first is based on the magnetic resonant near field coupling between two structures using relatively low frequencies $[1,2]$. This method has the inconvenient of allowing energy transfer over distances comparable to the size of the energy source (usually circular coils).

The second method consists of using high frequency (often above $1 \mathrm{GHz}$ ) electromagnetic waves for far-field energy transfer. It offers the advantage of allowing both short and long distance power transfer with relatively compact emitter and receiver sizes. High frequency waves are generated and captured by antennas and transformed into DC power by rectifying structures. The use of microwaves is more adapted in the case of medium to long range wireless powering of devices such as low power sensor networks or RFID tags [3, 4].

\section{RF-to-DC Rectifiers}

The association of a receiving antenna and an RF to DC rectifier circuit is usually called a rectenna ("rectifying antenna"). Rectennas are built using diodes or diode-mounted transistors for rectifying the high frequency sine wave generated by the antenna into DC voltage. Diodes are characterized by a threshold voltage that has to be overcome in order to put them in a conductive state. When important incident power levels are available, diode threshold voltage is not an issue, because incident voltage amplitude is much higher than the threshold voltage [5]. In the case of low incident power (below $1 \mathrm{~mW}$ ), loss in the diodes themselves become predominant. The choice was made to use zero bias Schottky diodes due to their low threshold (around $150 \mathrm{mV}$ ) and their low junction capacitance $(0.18 \mathrm{pF})$ [6].

Working frequency is also an important parameter to consider when designing a rectenna. It is often dictated by the desired application. At low frequencies (below $1 \mathrm{GHz}$ ), high gain antennas tend to be quite large. Increasing the frequency thus allows the use of more compact antennas. On the other hand, the amount of available power at a certain distance from an emitter is given by Friis equation:

$$
P_{r}=P_{t} \cdot G_{t} \cdot G_{r} \cdot\left(\frac{\lambda}{4 \pi R}\right)^{2}
$$


where $P_{t}$ is the power of the emitter, $G_{t}$ and $G_{r}$ are the emitter and receiver antenna gain, respectively, $\lambda$ is the wavelength used and $\mathrm{R}$ is the distance separating the emitter and the receiver. This means that available power at a certain distance from the emitter decreases as the frequency increases. Frequencies in the $1 \mathrm{GHz}-3 \mathrm{GHz}$ range are considered to provide a good compromise between free-space attenuation and antenna dimensions. We chose to design our circuits for a central frequency of $2.45 \mathrm{GHz}$.

Regardless of the topology used, a rectenna circuit can generally be presented as in Fig. 1.

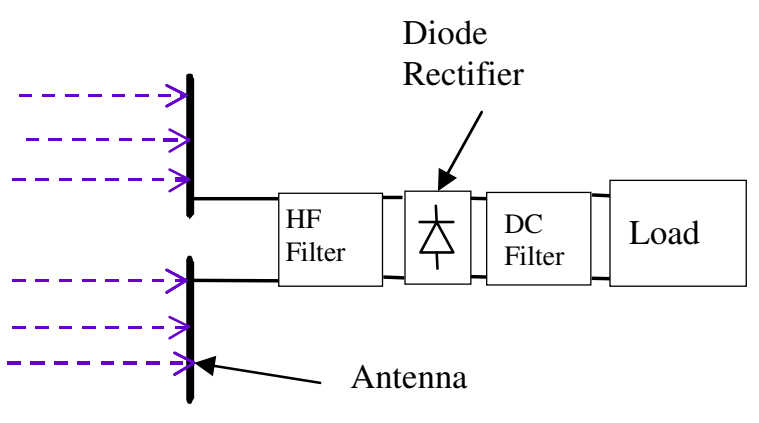

Fig. 1. Block diagram of rectenna circuit

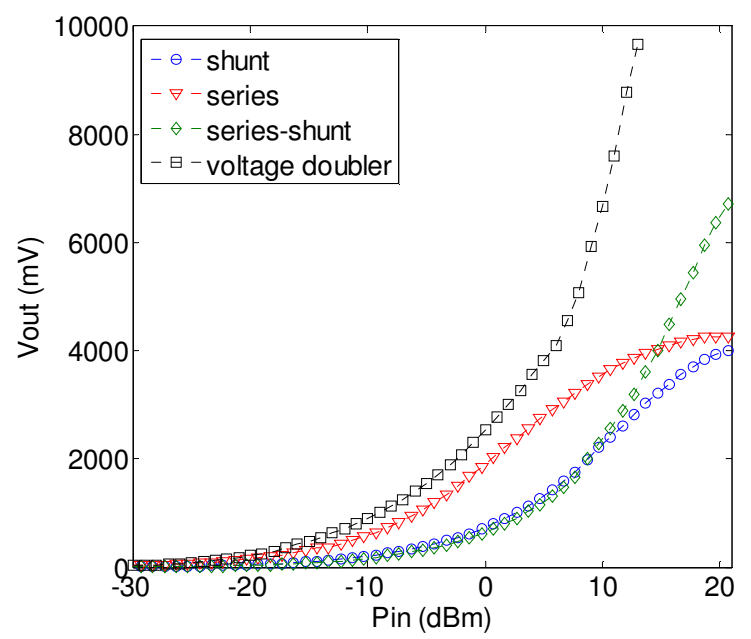

Fig. 2. Measured performance of the fabricated rectenna circuits

The incident RF power is captured by an antenna under the form of a high frequency sine wave. This is then transformed into DC power by the diode-based converter. A HF filter ensures impedance adaptation between the antenna and the diode rectifier for optimal power transfer. The output DC filter smoothens the output DC voltage and current by attenuating high frequency harmonics present in the RF signal or generated by the rectification process itself.

Standard bridge topology generally used in LF rectification has proven unsuitable to low power rectenna applications [6]. The topologies we used in this study are optimized for functioning with low input power levels. Two types of single-diode structures are presented: series mounted diode and shunt mounted diode. They theoretically offer the advantage of minimizing diode loss, which is proportional to the diode junction resistance.

Two different structures that theoretically offer the advantage of reaching higher DC voltage levels at the same input power level were also designed. A single stage and a two stage voltage doubler circuit have been designed and fabricated.

Prototypes were fabricated on a low-cost $1.6 \mathrm{~mm}$ FR4 substrate. Filters consist of high frequency and low-ESR passive components in 0402 SMD packaging. They were tested both stand-alone (RF power level is directly supplied by a power source through a SMA cable) as well as connected to an external antenna and supplied remotely.

Output DC voltage level is measured as a function of incident power level. Results are presented in Fig. 2. Shunt and single stage voltage doubler topologies have similar output voltages for power levels below $10 \mathrm{dBm}(10 \mathrm{~mW})$. The single stage voltage doubler provides higher DC voltage above $10 \mathrm{~mW}$. The two stage voltage doubler circuit excels in terms of output voltage. It provides $500 \mathrm{mV}$ at $-15 \mathrm{dBm}(30 \mu \mathrm{W})$ and reaches almost $10 \mathrm{~V}$ at $20 \mathrm{~mW}(13 \mathrm{dBm})$ of incident RF power. It's conversion efficiency is however the lowest of the four (less than $10 \%$ at $30 \mu \mathrm{W}$ and around $30 \%$ at $10 \mathrm{~mW}$ ). The single series mounted diode topology seems to offer the best compromise between output voltage level and conversion efficiency. 


\section{Wireless Energy Transfer Experiment}

The system was enlightened by a plane wave in order to evaluate the total amount of recoverable DC voltage as a function of the electric field strength, as shown in Fig. 3. We used a 9dBi horn antenna connected to the microwave signal generator as a microwave emitter and we placed the receiver at different distances from the emitter. The output power of the signal generator was set at $500 \mathrm{~mW}$. Energy is captured at receiver level via a 4 patch antenna array, with a measured gain of $9.4 \mathrm{dBi}$. The rectenna structure was the voltage doubler, as it has the highest detection sensitivity.

The output DC voltage of the receiver is measured and the results are presented in Fig. 4. Measurements show that relatively high output voltages are generated at important distance from the emitter $(0.4 \mathrm{~V}$ at $20 \mathrm{~m})$. The corresponding strength of the electric field at a distance of $20 \mathrm{~m}$ from the emitter is about $0.5 \mathrm{~V} / \mathrm{m}$. In terms of power density, this represents about $660 \mu \mathrm{W} / \mathrm{m}^{2}$. This confirms that usable DC electric power can be recovered from a relatively low microwave electric field using carefully designed RF/DC rectifiers and antenna arrays, under the condition that a DCDC boost converter is used for conditioning the available DC energy in order to reach the necessary voltage level for supplying an electronic circuit.

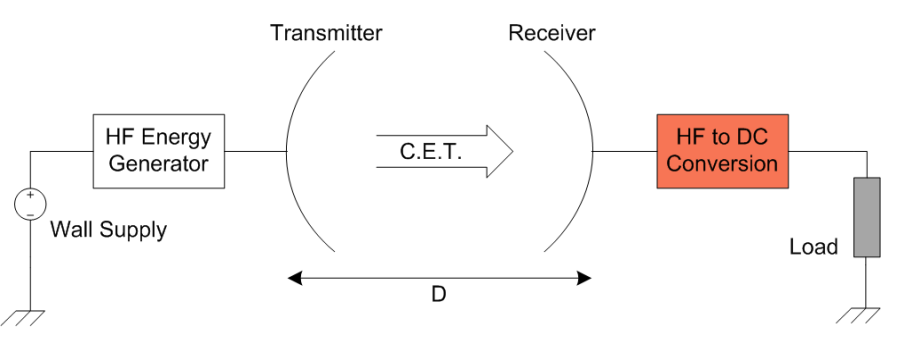

Fig. 3. Low power energy transfer experiment

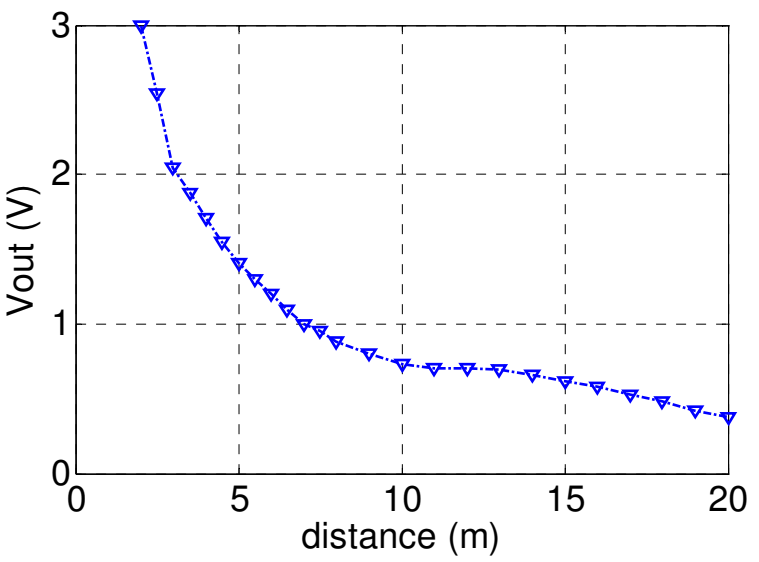

Fig. 4. Rectenna output DC voltage level

\section{MPPT system for Rectennas}

Experimental measurements show that rectennas can be modelled by a voltage source in series with its internal impedance [5, 6]. Fig. 5 shows I-V characteristics for a series-mounted diode rectenna topology, which are parallel lines. This proves that internal impedance of the rectenna is constant for a very wide range of input RF power. Fig. 6 confirms this hypothesis as rectenna efficiency has a maximum value for the same given output load, regardless of input power level.

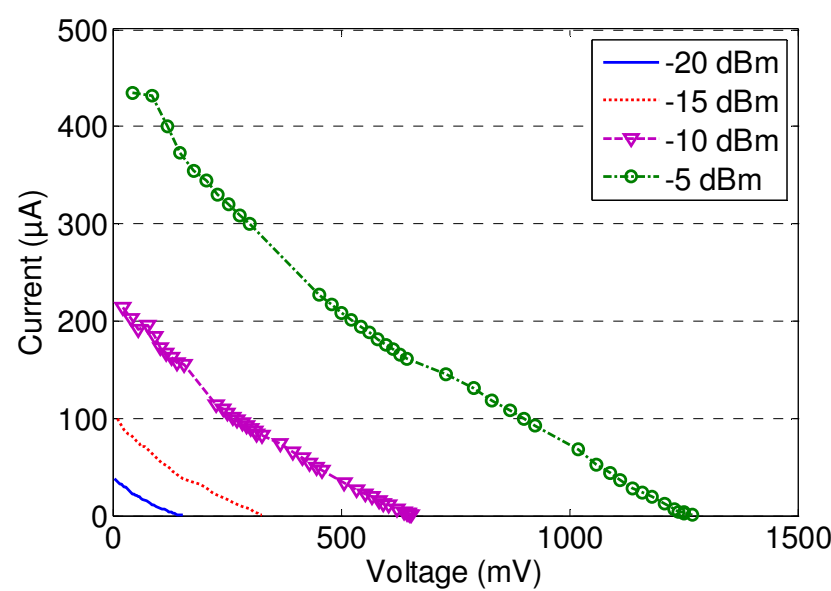

Fig. 5. I-V series rectenna characteristics for various values of input RF power

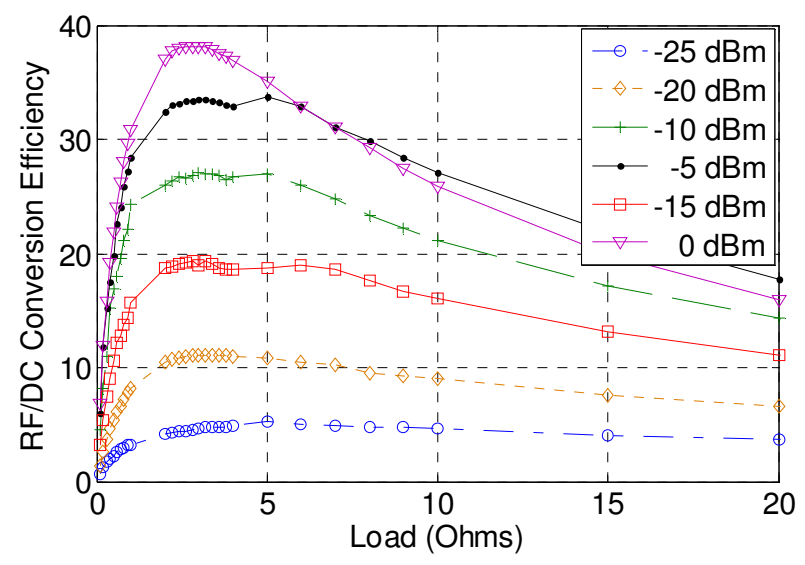

Fig. 6. Series rectennas efficiency depending on load 
The electric model of a rectenna is represented in Fig.8. For a given rectenna, Voltage source value is variable and depends on incident RF power. However, internal impedance is constant over a wide range of RF power.

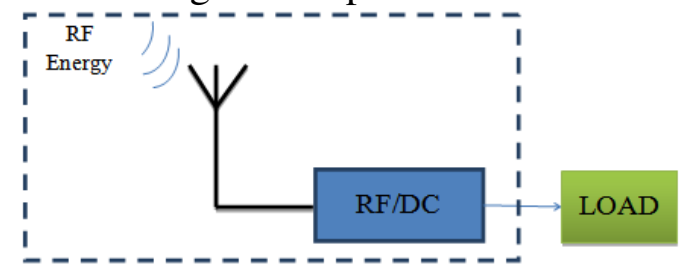

Fig. 7. Rectenna connected to a load

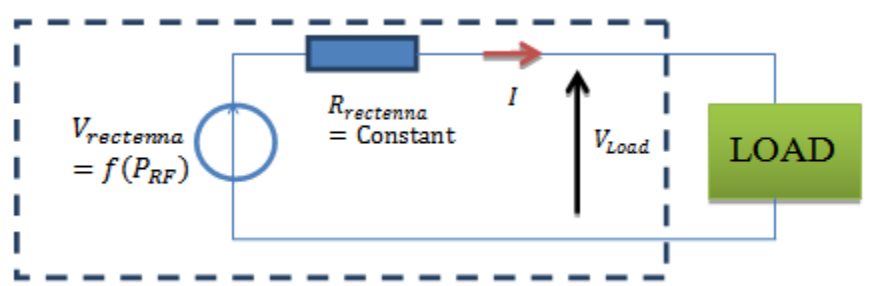

Fig. 8. Electric rectenna model

We extract maximum power from rectenna by loading it with an adapted load value (i.e. equal to the rectenna internal impedance. In practice, this is not easy to realize. That is why the use of MPPT (Maximum power point tracking) controller is necessary to accommodate a wide range of load values. MPPT controller is a technique widely used in high power systems (as photovoltaic and wind-powered systems), but seldom used in rectenna application. An example is given in [7]. The role of such system is to provide the necessary voltage level in order to supply the load and also to ensure impedance matching between source and load to ensure optimal power delivery.

\section{Conclusion}

This paper presents the general principle of wireless energy transmission using high frequency microwaves. It focuses mainly at receiver level, where the performances of several RF-to-DC conversion circuit topologies are compared. A wireless energy transmission experiment is also presented, and the perspective of a MPPT DC-DC boost converter designed for the low power levels specific to rectennas is discussed.

\section{References}

[1] A. Karalis, J. D. Joannopoulos, M. Soljacic, Efficient Wireless Non-Radiated Mid-Range Energy Transfert, Annals of Physics, 323 (2008) 34-48.

[2] A. P. Sample, D. a. Meyer, J. R. Smith, Analysis, Experimental Results, and Range Adaptation of Magnetically Coupled Resonators for Wireless Power Transfer, IEEE Transactions on Industrial Electronics, 58 (2011) 544-554.

[3] J. Yi, W. H. Ki, C. Y. Tsui, Analysis and design strategy of UHF Micro-Power CMOS Rectifiers for Micro-Sensor and RFID Applications, IEEE Transactions on Circuits and Systems, 54 (2007) 153-166.

[4] J.-w. Lee, B. Lee, A Long-Range UHF-Band Passive RFID Tag IC Based on High- Q Design Approach, IEEE Transactions on Industrial Electronics, 56 (2009) 2308-2316.

[5] B. Merabet, H. Takhedmit, B. Allard, L. Cirio, F. Costa, O. Picon, C. Vollaire, Low-cost converter for harvesting of microwave electromagnetic energy, IEEE Energy Conversion Congress and Exposition, San Jose, 2009.

[6] B. Merabet, F. Costa, H. Takhedmit, C. Vollaire, B. Allard, L. Cirio, O. Picon, A 2.45 GHz localized elements rectenna, 3rd IEEE International Symposium on Microwave, Antenna, Propagation and EMC Technologies for Wireless Communications, 2009.

[7] A. Dolgo, R. Zane, Z. Popovic, Power Management System for Online Low Power RF Energy Harvesting Optimization, IEEE Transactions on Circuits and Systems I: Regular Papers, 57 (2010) 1802-1811. 